\title{
HIGHLY CONNECTED MANIFOLDS OF POSITIVE $p$-CURVATURE
}

\author{
BORIS BOTVINNIK AND MOHAMMED LABBI
}

\begin{abstract}
We study and in some cases classify highly connected manifolds which admit a Riemannian metric with positive $p$-curvature. The $p$-curvature was defined and studied by the second author in earlier papers. It turns out that the positivity of $p$-curvature is preserved under surgeries of codimension at least $p+3$. This gives a key to reducing a geometrical classification problem to a topological one, in terms of relevant bordism groups and index theory. In particular, we classify 3-connected manifolds with positive 2-curvature in terms of the bordism groups $\Omega_{*}^{\text {spin }}, \Omega_{*}^{\text {string }}$, and by means of an $\alpha$-invariant and a Witten genus $\phi_{W}$. Here we use results from Anand Dessai (2009), which provide appropriate generators of the ring $\Omega_{*}^{\text {string }} \otimes \mathbf{Q}$ in terms of "geometric $\mathbb{C a P}^{2}$-bundles", where the Cayley projective plane $\mathbb{C a P}^{2}$ is a fiber and the structure group is $F_{4}$ which is the isometry group of the standard metric on $\mathrm{CaP}^{2}$.
\end{abstract}

\section{Contents}

1. Introduction and statement of the main results

1.1. Positive scalar curvature

1.2. Positive $p$-curvature

1.3. Geometric $\mathbf{H} \mathbf{P}^{2}$-bundles

1.4. Main results

1.5. Generalizations

1.6. Plan of the paper

2. String and $B O\langle\ell\rangle$-cobordism: Basic definitions

3. Surgeries and $B O\langle\ell\rangle$-manifolds

3.1. Non-string 3-connected manifolds

3.2. Non-Fivebrane 7-connected manifolds

4. Positive curvature and surgeries

4.1. Positive $p$-curvature

4.2. Positive second Gauss-Bonnet curvature

5. First applications: Proof of Theorems A, B and B'

5.1. Proof of Theorem A

5.2. Proof of Theorems B and $\mathrm{B}^{\prime}$

5.3. Genera for string manifolds and positive curvature

6. Witten genus and its kernel in $\Omega_{*}^{\text {string }} \otimes \mathbf{Q}$

6.1. Cayley projective plane 


\section{InTRODUCTION AND STATEMENT OF THE MAIN RESUlts}

1.1. Positive scalar curvature. There is a fundamental result due to Gromov and Lawson [6] and Schoen and Yau [17] known as "Surgery Theorem". It shows that positivity of the scalar curvature can be preserved under surgery of codimension at least three. In particular, the surgery technique provides a key to classifying simply connected manifolds admitting a metric with positive scalar curvature, 6], [18. These are the results:

Theorem 1.1 ([6, Theorem A]). Let $M$ be a compact non-spin simply connected manifold with $\operatorname{dim} M=n \geq 5$. Then $M$ always admits a metric $g$ with positive scalar curvature.

Let $\alpha: \Omega_{n}^{\text {spin }} \rightarrow K O_{n}$ be the Atiyah-Bott-Shapiro homomorphism which evaluates the index of the Dirac operator on a spin manifold $M$ representing a cobordism class $[M] \in \Omega_{n}^{\text {spin }}$.

Theorem 1.2 ([6, Theorem B], [18]). Let $M$ be a compact spin simply connected manifold with $\operatorname{dim} M=n \geq 5$. Then $M$ admits a metric $g$ with positive scalar curvature if and only if $\alpha([M])=0$ in the group $K O_{n}$.

It turns out that there are many other Riemannian invariants that are also stable under some type of surgeries; see, for example, 1, 4, 13, 14, 20. Among such invariants are the $p$-curvature $s_{p}$ and the second Gauss-Bonnet curvature which were studied by the second author; see [8,11].

1.2. Positive $p$-curvature. Let $(M, g)$ be a Riemannian manifold, and $T M$ be the tangent bundle. We denote by $G_{p}(T M)$ the bundle of Grassmanians of $p$ dimensional subspaces of the tangent bundle $T M$. Then the $p$-curvature $s_{p}$ is defined as a function $s_{p}: G_{p}(T M) \rightarrow \mathbf{R}$ as follows. For a $p$-dimensional space $V \in G_{p}\left(T M_{x}\right)$, the value of $s_{p}(V)$ is a "partial trace" of the curvature tensor, along all directions, perpendicular to the subspace $V \subset T M_{x}$; see 8 and section 5 for details. The curvature $s_{0}$ is nothing but the scalar curvature Scal; furthermore, the function $s_{p-1}$ can be thought of as an appropriate trace of $s_{p}$. In particular, positivity of $s_{p}$ implies positivity of the curvatures $s_{j}$ for all $j<p$, including the scalar curvature. It turns out that positivity of the $p$-curvature $s_{p}$ is also stable under surgeries of codimension at least $3+p$; see [8, Main Theorem].

The surgery result [8, Main Theorem] gives an appropriate setup to classifying manifolds admitting a metric with positive $p$-curvature for $p \geq 1$ similar to the case of positive scalar curvature. The first interesting case is when $p=1$. Then the curvature $s_{1}$ coincides (up to a factor 2) with the quadratic form associated to the 2 -tensor $S$ defined by

$$
S_{i j}=\frac{1}{2} \mathrm{Scal} \cdot g_{i j}-\mathrm{Ric}_{i j} .
$$

The tensor $-S$ is also known as the Einstein tensor, and the 1-curvature $s_{1}$ is called the Einstein curvature; see [8] and [9]. We notice that

$$
\operatorname{tr} S=\frac{(n-2)}{2} \text { Scal . }
$$

Thus positivity of the tensor $S$ is the same as positivity of the curvature $s_{1}$, and positivity of $s_{1}$ implies positivity of the scalar curvature for $n \geq 3$. 
An interesting case here is when the manifolds in question are 2-connected. Then such manifolds are necessarily spin-manifolds, and the relevant cobordism group is $\Omega_{n}^{\text {spin }}$. Here is the classification result analogous to Theorem 1.2.

Theorem 1.3 ([8, Theorem I]). Let $M$ be a compact 2-connected manifold with $\operatorname{dim} M=n \geq 7$. Then $M$ admits a metric $g$ with positive 1-curvature if and only if $\alpha([M])=0$ in the group $K O_{n}$.

The main technique in proving Theorem 1.3 is a Surgery Theorem [8, Main Theorem] and the results by S. Stolz on geometric $\mathbf{H P}^{2}$-bundles.

1.3. Geometric $\mathbf{H P}^{2}$-bundles. We recall that in order to prove that vanishing of the index $\alpha([M]) \in K O_{n}$ is sufficient for the existence of a metric with positive scalar curvature on $M$, S. Stolz proves that all cobordism classes in ker $\alpha \subset \Omega_{n}^{\text {spin }}$ can be realized as total spaces of geometric $\mathbf{H P}^{2}$-bundles.

In more detail, let $P S p(3)$ be the projectivization of the symplectic orthogonal group $S p(3)$. It is well known that the group $P S p(3)$ is the isometry group of the standard metric on the projective plane $\mathbf{H P}^{2}$. Let $B P S p(3)$ be the classifying space of the group $P S p(3)$, and $E P S p(3) \rightarrow B P S p(3)$ be the universal principal bundle. This gives a universal geometric $\mathbf{H P}^{2}$-bundle $E\left(\mathbf{H P}^{2}\right) \rightarrow B P S p(3)$ with a fiber $\mathbf{H} \mathbf{P}^{2}$ and a structure group $P S p(3)$, where the total space $E\left(\mathbf{H P}^{2}\right)$ is defined in a usual way:

$$
E\left(\mathbf{H P}^{2}\right)=E P S p(3) \times_{P S p(3)} \mathbf{H P}^{2} .
$$

Then for any map $f: B \rightarrow B P S p(3)$, there is a natural pull-back $\mathbf{H P}^{2}$-bundle $E \rightarrow B$ given by the diagram

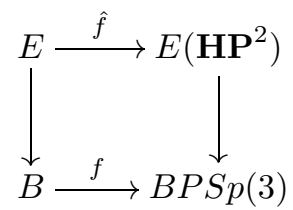

This construction defines a transfer map

$$
T: \Omega_{n-8}^{\text {spin }}(B P S p(3)) \longrightarrow \Omega_{n}^{\text {spin }}
$$

which takes a cobordism class of a map $f: B \rightarrow B P S p(3)$ to the cobordism class of the manifold $E$ as above. The following result provides a key in proving the necessity in Theorem 1.2 .

Theorem 1.4 (Stolz, [18]). There is an isomorphism $\operatorname{Im} T \cong \operatorname{ker} \alpha$.

By construction, the total space $E$ of a geometric $\mathbf{H P}^{2}$-bundle carries a metric with positive scalar curvature, which is obtained by a choice of any metric on the base and giving a standard homogeneous metric to each fiber $\mathbf{H P}^{2}$ scaled appropriately to get a positive scalar curvature on $E$.

One can observe that if $E$ is the total space of a geometric $\mathbf{H} \mathbf{P}^{2}$-bundle, then it carries a metric with positive curvature $s_{p}$ for $p \leq 6$.

1.4. Main results. Assume that $M$ is a 3 -connected manifold. Then $M$ has a canonical spin-structure. There are two possibilities: either $M$ is a string manifold or not. It is well known that the obstruction to existence of a string-structure is 
given by $\frac{1}{2} p_{1}(M)$, where $p_{1}$ is the first Pontryagin class. The following result is somewhat analogous to Theorem 1.1 .

Theorem A. Let $M$ be a compact 3-connected non-string manifold with $\operatorname{dim} M=$ $n \geq g$. Then $M$ admits a Riemannian metric $g$ with positive 2-curvature if and only if $\alpha([M])=0$ in the group $K O_{n}$, where $\alpha: \Omega_{n}^{\text {spin }} \rightarrow K O_{n}$ is as above.

Any 3 -connected manifold is spin, and $\Omega_{*}^{\text {spin }}$ is a relevant bordism group here. We use the surgery technique and Theorem 1.4 to show that if $\alpha([M])=0$ and $M$ is not string, then it has a metric with positive 2-curvature which is "pulled back" from a nice metric on the total space of a geometric $\mathbf{H P}^{2}$-bundle as above.

Now let $M$ be 3 -connected and string. A relevant bordism group here is $\Omega_{*}^{\text {string }}$. Precisely, we prove the following theorem which is analogous to Theorem B of [6].

Theorem B. Let $M_{1}$ be a compact $(3+r)$-connected, $0 \leq r \leq 3$, string manifold of dimension $n \geq 9+2 r$. Assume that $\left[M_{1}\right]=\left[M_{0}\right]$ in the cobordism group $\Omega_{n}^{\text {string }}$, where $M_{0}$ admits a metric $g_{0}$ with $s_{r+2}\left(g_{0}\right)>0$. Then $M_{1}$ also admits a metric $g_{1}$ with $s_{r+2}\left(g_{1}\right)>0$.

In particular, a compact 3-connected string manifold $M$ of dimension $n \geq 9$ that is string cobordant to a manifold of positive 2-curvature admits a metric with positive 2-curvature.

For instance, if $M$ is string cobordant to zero, then the conclusion of the theorem holds for $M$. It is known that $\Omega_{n}^{\text {string }}=0$ for $n=11$ or $n=13$; therefore any compact 3-connected string manifold of dimension 11 or 13 always has a metric with positive 2-curvature.

Let $I$ denote the subset of $\Omega_{*}^{\text {string }}$ which consists of bordism classes containing representatives with positive 2-curvature. Clearly $I$ is an ideal of $\Omega_{*}^{\text {string }}$ since the cartesian product of a manifold of positive 2-curvature with an arbitrary manifold has positive 2-curvature. We therefore define the following geometrical genus:

$$
\Pi: \Omega_{*}^{\text {string }} \rightarrow \Omega_{*}^{\text {string }} / I,
$$

which is a ring homomorphism.

Let $\phi_{W}: \Omega_{*}^{\text {string }} \rightarrow \mathbf{Z}[[q]]$ be the Witten genus; see [5, 19], and section 6 below. By definition, if $\phi_{W} x \neq 0$, then $x \in \Omega_{*}^{\text {string }}$ has infinite order. We prove the following result which is analogous to Corollary B of [6].

Corollary B. Let $N$ be a $(3+r)$-connected, for $0 \leq r \leq 3$, string manifold of dimension at least $9+2 r$ with vanishing Witten genus. Then some multiple $N \sharp \cdots \sharp N$ carries a metric of positive $(r+2)$-curvature.

In particular, if $N$ is a 3-connected string manifold of dimension at least 9 with vanishing Witten genus, then some multiple $N \sharp \cdots \sharp N$ carries a metric of positive 2-curvature.

This result suggests that the geometric genus $\Pi$ is related to the Witten genus. It is an open question whether $N$ itself carries a metric of positive 2-curvature.

Clearly, Theorems A, B and Corollary B give only partial classification of manifolds with metrics of positive 2-curvature. However, we use a construction which eventually may be useful in obtaining an affirmative classification. Before stating our conjecture, we briefly describe the construction.

Let $F_{4}$ be the 52 -dimensional compact simple sporadic Lie group. It is well known that it contains a closed subgroup isomorphic to $\operatorname{Spin}(9)$ which is unique up to inner 
automorphism. We denote by $\mathbb{C a P}^{2}$ the Cayley projective plane which coincides with the homogeneous space $F_{4} / \operatorname{Spin}(9)$. Then the canonical homogeneous metric on $\mathbb{C a P}^{2}$ has $F_{4}$ as a full isometry group; see [23, p. 264]. Let $B F_{4}$ be a classifying space, and $E F_{4} \rightarrow B F_{4}$ be a universal principle $F_{4}$-bundle. A universal geometrical $\mathbb{C a P}^{2}$-bundle can be identified with the fiber bundle $B \operatorname{Spin}(9) \rightarrow B F_{4}$ which has a fiber $\mathbb{C a P}^{2}$ and a structure group $F_{4}$. Then for a manifold $L$ and a map $f: L \rightarrow$ $B F_{4}$, we obtain the following map of fiber bundles:

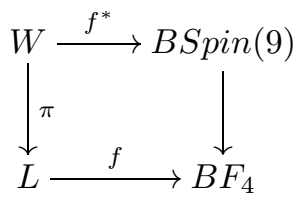

The fiber bundle $\pi: W \rightarrow L$ as above is called a geometrical $\mathbb{C a P}^{2}$-bundle.

It is well known that $\Omega_{*}^{\text {string }} \otimes \mathbf{Q}$ is a polynomial ring. In more detail, A. Dessai shows that there exist generators $x_{4 k}$, such that

$$
\Omega_{*}^{\text {string }} \otimes \mathbf{Q} \cong \mathbf{Q}\left[x_{8}, x_{12}, x_{16}, \ldots\right],
$$

and each element $x_{4 k}$ with $k \geq 4$ is represented by a manifold $W^{4 k}$ which is a total space of a geometrical $\mathbb{C a P}^{2}$-bundle $\pi_{k}: W^{4 k} \rightarrow L^{4 k-16}$; see [5] and section 6] below. We consider a transfer map

$$
T^{\text {string }}: \Omega_{\ell}^{\text {string }}\left(B F_{4}\right) \longrightarrow \Omega_{\ell+16}^{\text {string }}
$$

given as follows. Let $f: L \rightarrow B F_{4}$ be a map representing an element $x \in$ $\Omega_{\ell}^{\text {string }}\left(B F_{4}\right)$. Then the manifold $W$ from (1.1) represents the element $T^{\text {string }}(x) \in$ $\Omega_{\ell+16}^{\text {string }}$. Also, we recall that there is an integral version of the Witten genus

$$
\phi_{W}^{\mathbf{Z}}: \Omega_{*}^{\text {string }} \rightarrow K O_{*}[[q]]
$$

which factors through the coefficients $\mathrm{tmf}_{*}$ of the topological modular forms theory tmf (formally known as $e o_{2}$ ):

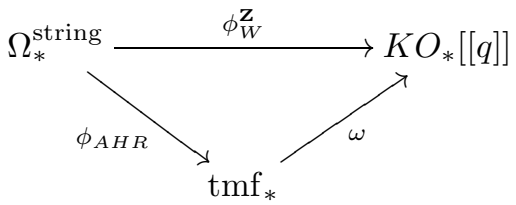

Here $\phi_{A H R}: \Omega_{*}^{\text {string }} \rightarrow \operatorname{tmf}_{*}$ is the string-orientation constructed by Ando, Hopkins and Strickland; see [2, 3 .

Remark 1.5. It is known that the groups $\Omega_{*}^{\text {string }}$ have no $p$-torsion away from $p=$ 2,3 . It is tempting to conjecture that $\operatorname{Im} T^{\text {string }}$ and $\operatorname{Ker} \phi_{A H R}$ coincide in $\Omega_{*}^{\text {string }}$ localized at primes 2 and 3. It turns out that this is too optimistic: the authors were informed by M. Joachim that the image $\operatorname{Im} T^{\text {string }}$ is strictly less than Ker $\phi_{A H R}$ in dimension 32. Nevertheless, we think that one may use other homogeneous spaces, besides $\mathbb{C a P}^{2}$, to represent elements of the kernel Ker $\phi_{A H R}$ by manifolds with positive 2-curvature.

Conjecture C. Let $M$ be a 3-connected string manifold with $\operatorname{dim} M=n \geq 9$. Then $M$ admits a Riemannian metric of positive 2-curvature if and only if $\phi_{A H R}([M])=$ 0 in $\operatorname{tmf}_{n}$. 
We note that Conjecture $\mathrm{C}$ is weaker than Stolz' conjecture [19, Conjecture 1.1] on the existence of a metric with positive Ricci curvature. However it seems that it is still very difficult to verify.

1.5. Generalizations. The previous results are generalized in this paper in different directions.

On one hand, we show that all the previous theorems and conjectures are still valid if one replaces the everywhere positive 2-curvature $s_{2}$ by the positive second Gauss-Bonnet curvature $h_{4}$ or by both $s_{2}>0$ and $h_{4}>0$. Recall that the $h_{4}$ curvature is a scalar function defined on the manifold that generalizes the usual scalar curvature. It is shown in 11 that it is preserved under surgeries of codimension at least 5 .

On the other hand, we prove that similar results hold for 3-curvature $s_{3}$ in the frame of 4-connected Fivebrane and non-Fivebrane manifolds. Recall that a Fivebrane manifold is a string manifold for which the fractional pontryagin class $\frac{1}{6} p_{2}$ vanishes.

The above Corollary $\mathrm{B}$ asserts in particular that if a compact 6-connected manifold $N$ is with dimension $\geq 15$ and with vanishing Witten genus, then some multiple of it $N \sharp \cdots \sharp N$ carries a metric of positive 5 -curvature. We prove the following analogous of Theorem A in this context:

Theorem $\mathbf{A}^{\prime}$. Let $N$ be a 7-connected and non-Fivebrane compact manifold of dimension $\geq 15$. If $N$ is string-cobordant to a manifold $M$ which carries a metric with positive 6 -curvature, then $N$ also carries a metric with positive 6 -curvature.

In particular, if a compact non-Fivebrane 7-connected manifold $N$ of dimension $\geq 15$ has a vanishing Witten genus, then some multiple of it $N \sharp \cdots \sharp N$ carries a metric of positive 6-curvature.

It remains an open question to prove that $N$ itself carries a metric of positive 6-curvature.

From another prospective, we prove the following generalization of Theorem B:

Theorem $\mathbf{B}^{\prime}$. Let $M_{1}$ be a compact $(4+r)$-connected, $0 \leq r \leq 3$, Fivebrane manifold of dimension $n \geq 11+2 r$. Assume that $\left[M_{1}\right]=\left[M_{0}\right]$ in the cobordism

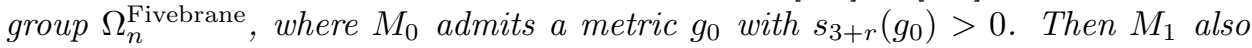
admits a metric $g_{1}$ with $s_{3+r}\left(g_{1}\right)>0$.

In particular, a compact 4-connected Fivebrane manifold $M$ with $\operatorname{dim} M=n \geq$ 11 that is Fivebrane cobordant to a manifold of positive 3-curvature also carries a metric with positive 3-curvature.

This paper also contains further generalizations of the previous results, whenever appropriate, to all higher $p$-curvatures in the case of highly connected $B O\langle\ell\rangle$ manifolds.

1.6. Plan of the paper. Section 2 contains basic definitions of string and Fivebrane manifolds, string and Fivebrane cobordism rings and more general $B O\langle\ell\rangle$ manifolds and the corresponding cobordism rings.

In sections 3 and 4 , we prepare for the proof of the main results. In section 3 , we study some interactions between the codimension size of a surgery made within a given $B O\langle\ell\rangle$-cobordism class and the order of connectivity of representatives of that class. In section 4 , we recall the definitions of $p$-curvatures $s_{p}$ and the second 
Gauss-Bonnet curvature $h_{4}$. We emphasize that the most important property of these curvatures is the stability of their positivity under surgeries of sufficiently high codimensions.

In section 5, we prove Theorems $\mathrm{A}, \mathrm{B}$ and $\mathrm{B}^{\prime}$. In section 6 we recall useful material about the Witten genus and the recent results of $\mathrm{A}$. Dessai about the rational cobordism groups and the kernel of the Witten genus. The results of section 6 are used in section 7 in proving Theorem $\mathrm{A}^{\prime}$ and Corollary B.

\section{String And $B O\langle\ell\rangle$-COBordism: Basic Definitions}

Let $\mathbf{R}^{n+k}$ be the Euclidian space. We denote by $G_{n}\left(\mathbf{R}^{n+k}\right)$ the Grassmanian manifold of $n$-dimensional subspaces of $\mathbf{R}^{n+k}$, and by

$$
U_{k, n} \longrightarrow G_{n}\left(\mathbf{R}^{n+k}\right) \quad \text { and } \quad U_{k, n}^{\perp} \longrightarrow G_{n}\left(\mathbf{R}^{n+k}\right)
$$

the tautological bundle and its orthogonal complement respectively. Then one obtains the spaces

$$
B O(n):=\lim _{k} G_{n}\left(\mathbf{R}^{n+k}\right) \quad \text { and } \quad B O:=\lim _{n} B O(n),
$$

which are the classifying spaces of the orthogonal group $O(n)$ and its stable version $O:=\lim _{n} O(n)$. The homotopy groups of $B O$ are well known:

$$
\pi_{q} B O=\left\{\begin{array}{cl}
\mathbf{Z} / 2 & \text { if } q=1,2(\bmod 8) \\
\mathbf{Z} & \text { if } q=0,4(\bmod 8) \\
0 & \text { else }
\end{array}\right.
$$

Consider the Postnikov tower of the space $B O$ :

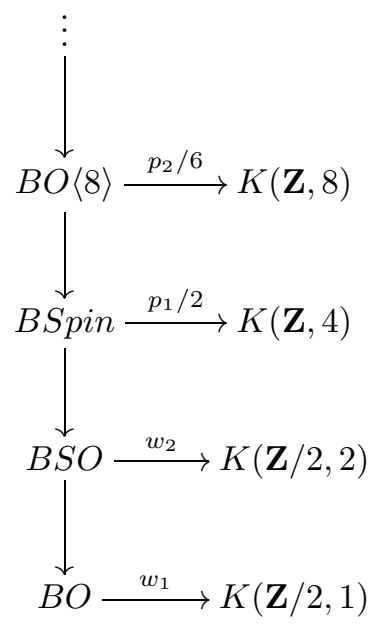

In each step the lowest homotopy group is killed by the map into the corresponding Eilenberg-MacLane space, and $w_{1}, w_{2}$ are the Stiefel-Whitney classes and $p_{1}, p_{2}$ are the Pontryagin classes respectively.

Now let $M$ be a manifold, $\operatorname{dim} M=n$. We denote by $h_{0}$ the Euclidian metric on $\mathbf{R}^{n+k}$. Then an embedding $j: M \hookrightarrow \mathbf{R}^{n+k}$ provides $M$ with the Riemannian metric $g=j^{*} h_{0}$ induced from the Euclidian space $\mathbf{R}^{n+k}$. Furthermore, the metric 
$g$ gives the tangent and normal bundles $T M$ and $N M$ the Euclidian structure, in particular, we have the Gauss map

$$
\bar{f}: M \longrightarrow G_{k}\left(\mathbf{R}^{n+k}\right)
$$

such that $\bar{f}^{*} U_{k, n}=N M$ and $\bar{f}^{*} U_{k, n}^{\perp}=T M$. A homotopy class of $\bar{f}$ depends on the embedding $j: M \hookrightarrow \mathbf{R}^{n+k}$; however, it determines uniquely a homotopy class of the composition

$$
f: M \stackrel{\bar{f}}{\longrightarrow} G_{k}\left(\mathbf{R}^{n+k}\right) \longrightarrow B O .
$$

We say that a manifold $M$ has a string-structure if the Gauss map $f: M \longrightarrow B O$ lifts to the map $\hat{f}: M \longrightarrow B O\langle 8\rangle$, i.e. the following diagram commutes:

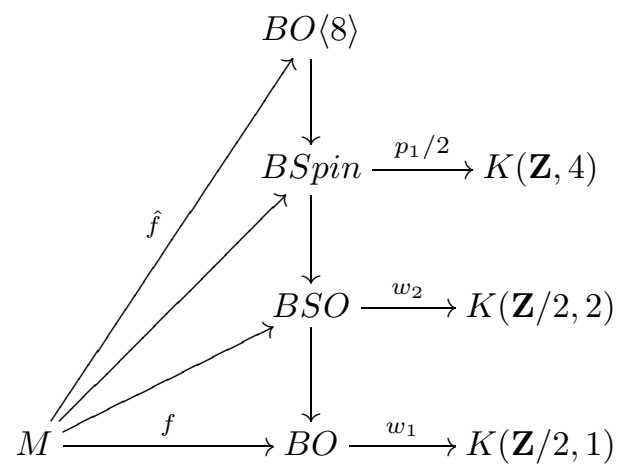

A choice of the lift $\hat{f}$ is sometimes called a string-structure on $M$. We emphasize that usually we use a string-structure on the normal bundle $N M$; this implies that the tangent bundle $T M$ also has a string-structure. We denote by $\Omega_{n}^{\text {string }}$ the corresponding cobordism group.

This construction has a more general setting. Let $B O\langle\ell\rangle$ be the $(\ell-1)$-connected cover of $B O$. We say that a manifold $M$ has $B O\langle\ell\rangle$-structure if it is given a lift $f_{\langle\ell\rangle}$ of the standard Gauss map as above. Then there is a corresponding cobordism group $\Omega_{n}^{\langle\ell\rangle}$. Clearly we have that $\Omega_{n}^{\langle 4\rangle}=\Omega_{n}^{\text {spin }}$, and $\Omega_{n}^{\langle 8\rangle}=\Omega_{n}^{\text {string }}$. There is one more special case when manifolds have $B O\langle 9\rangle$-structure: these are string manifolds with the vanishing class $\frac{1}{6} p_{2}$. In some papers, for instance see [16], manifolds with $B O\langle 9\rangle$-structure are called Fivebrane manifolds, and the cobordism group $\Omega_{n}^{\langle 9\rangle}$ is called the Fivebrane cobordism and denoted as $\Omega_{n}^{\text {Fivebrane }}$.

\section{SuRgeries AND $B O\langle\ell\rangle$-MANIFOLDS}

Let $M$ be a closed $n$-manifold, $S^{s} \subset M$ be an embedded sphere with trivial normal bundle and let $t=n-s-1$. This gives an embedding $S^{s} \times D^{t+1} \subset M$ which extends the embedding $S^{s} \subset M$. Then a surgery along the embedded $S^{s} \subset M$ gives the manifold

$$
M^{\prime}=\left(M \backslash\left(S^{s} \times D^{t+1}\right)\right) \cup_{S^{s} \times S^{t}}\left(D^{s+1} \times S^{t}\right) .
$$

Let $x \in \pi_{s}(M)$ be an element represented by a map $\xi: S^{s} \rightarrow M$. If $2 s<n$, then according to the Whitney Embedding Theorem, the map $\xi$ can be deformed to an embedding $S^{s} \subset M$. Then we say that the element $x \in \pi_{s}(M)$ can be killed by a surgery if such an embedding has trivial normal bundle. 
Let $f: M \rightarrow B O$ be the map classifying the stable normal bundle of $M$; it gives the induced homomorphism $f_{*}: \pi_{s}(M) \rightarrow \pi_{s}(B O)$. The following result is well known (see [15, Corollary 5.64], for example):

Lemma 3.1. Let $M$ be a smooth manifold and let $f: M \rightarrow B O$ be the map classifying the stable normal bundle of $M$. Assume $2 s<n=\operatorname{dim} M$. Then an element $x \in \pi_{s}(M)$ can be killed by a surgery if and only if $f_{*}(x)=0$ in $\pi_{s}(B O)$.

The previous lemma implies in particular that for a $B O\langle\ell\rangle$-manifold $M$ of dimension $n>2 s$, every map $S^{s} \rightarrow M$ has trivial normal bundle for $s \leq l-1$. We have therefore proved the following lemma:

Lemma 3.2. Assume $n>2(\ell-k) \geq 2$ and $k \geq 1$.

(1) Let $x \in \Omega_{n}^{\langle\ell\rangle}$. Then $x$ can be represented by some $(\ell-k)$-connected manifold.

(2) Let $M_{0}$ and $M_{1}$ be $(\ell-k)$-connected $B O\langle\ell\rangle$-manifolds. Then if $M_{0}$ and $M_{1}$ represent the same element $x \in \Omega_{n}^{\langle\ell\rangle}$, there exists a BO$\langle\ell\rangle$-cobordism $\left(W, M_{0}, M_{1}\right)$, where the pair $\left(W, M_{1}\right)$ is $(\ell-k)$-connected.

In particular, if $n>8$, any element $x \in \Omega_{n}^{\text {string }}$ can be represented by a 4 connected manifold, and if $M_{0}, M_{1}$ are two 4-connected manifolds representing $x$, then there exists a string-cobordism $\left(W, M_{0}, M_{1}\right)$ where $\left(W, M_{1}\right)$ is 4 -connected.

Next, we need more details on bordisms between $B O\langle\ell\rangle$-manifolds. We start with the following fact from basic Morse theory:

Lemma 3.3. Let $\left(W, M_{0}, M_{1}\right)$ be a simply connected bordism, $\operatorname{dim} W=n+1$, and let $n \geq p+3$, where $p$ is a positive integer. Assume $H_{j}\left(W, M_{1} ; \mathbf{Z}\right)=0$ for all $j \leq p$; then $M_{1}$ can be obtained from $M_{0}$ by surgeries of codimension at least $p+1$.

The following result is a consequence of Lemma 3.3 .

Proposition 3.4. Let $M_{1}$ be a compact $r$-connected $B O\langle\ell\rangle$-manifold of dimension $n$, where $n \geq 2 r+3$ and $\ell \geq r+2$. Let $M_{0}$ be a compact manifold, such that $\left[M_{0}\right]=\left[M_{1}\right]$ in $\Omega_{n}^{\langle\ell\rangle}$. Then $M_{1}$ can be obtained from $M_{0}$ by surgeries of codimension at least $r+2$.

Proof. Let $\left(W, M_{0}, M_{1}\right)$ be a $B O\langle\ell\rangle$-cobordism, and let $M_{1}$ be $r$-connected. Using surgeries we can assume that $W$ is $(r+1)$-connected since $r+1 \leq \ell-1$ and the dimension of $W$ is sufficiently high. Consequently, we have $H_{i}(W)=0$ for all $i \leq r+1$. On the other hand $M_{1}$ is $r$-connected; thus $H_{i}\left(M_{1}\right)=0$ for all $i \leq r$ and therefore $H_{i}\left(W, M_{1}\right)=0$ for all $i \leq r+1$. Finally, Lemma 3.3 implies that $M_{1}$ can be obtained from $M_{0}$ by surgeries of codimension $r+2$.

Remarks 3.1. (1) For $r=1$ and $\ell=4$, Proposition 3.4 asserts the following: if $M_{1}$ is a compact simply connected spin manifold of dimension $n \geq 5$ that is spincobordant to a manifold $M_{0}$, then $M_{1}$ can be obtained from $M_{0}$ by surgeries of codimension at least three. This was first noticed by Gromov and Lawson; see [6].

(2) For $r=2$ and $\ell=4$, Proposition 3.4 is the same as the surgery Lemma 4.2 in [8]: if $M_{1}$ is a compact 2-connected manifold of dimension $n \geq 7$ that is spin-cobordant to a manifold $M_{0}$, then $M_{1}$ can be obtained from $M_{0}$ by surgeries of codimension $\geq 4$.

We specify Theorem 3.4 for string manifolds. 
Corollary 3.5. Let $M_{1}$ be a compact $r$-connected string manifold of dimension $n \geq 2 r+3$, where $r \leq 6$. Then if $M_{0}$ is a compact manifold such that $\left[M_{0}\right]=\left[M_{1}\right]$ in $\Omega_{n}^{\text {string }}$, then $M_{1}$ can be obtained from $M_{0}$ by surgeries of codimensions at least $r+2$.

In particular, if $M_{1}$ is a compact 3-connected string manifold of dimension $n \geq 9$ string-cobordant to a manifold $M_{0}$, then $M_{1}$ can be obtained from $M_{0}$ by surgeries of codimensions at least 5 .

If we continue our climbing of the Postnikov tower we reach the $B O\langle 9\rangle$-manifolds or Fivebrane manifolds. It is well known that the corresponding Postnikov invariant is given by $\frac{1}{6} p_{2}$, where $p_{2}$ is the second Pontryagin class. We specify Proposition 3.4 for Fivebrane manifolds:

Corollary 3.6. Let $M_{1}$ be an $r$-connected Fivebrane manifold of dimension $n \geq$ $2 r+3$, where $r \leq 7$. Then if $M_{0}$ is a Fivebrane manifold with $\left[M_{0}\right]=\left[M_{1}\right]$ in $\Omega_{n}^{\text {Fivebrane }}$, then $M_{1}$ can be obtained from $M_{0}$ by surgeries of codimensions at least $r+2$.

In particular, if $M_{1}$ is a compact 4-connected Fivebrane manifold of dimension $n \geq 11$ that is Fivebrane cobordant to a manifold $M_{0}$, then $M_{1}$ can be obtained from $M_{0}$ by surgeries of codimensions at least 6 .

3.1. Non-string 3-connected manifolds. In contrast with the result in Corollary 3.5, we prove the following result for 3-connected but non-string manifolds.

Proposition 3.7. Let $M_{1}$ be a 3-connected and non-string compact manifold of dimension $\geq g$. If $M_{1}$ is spin cobordant to a manifold $M_{0}$, then $M_{1}$ can be obtained from $M_{0}$ by surgeries of codimension $\geq 5$.

Proof. Let $\left(W, M_{0}, M_{1}\right)$ be a spin cobordism, where $M_{1}$ is 3-connected, non-string with dimension at least 7 , and $W$ is spin. Using surgeries we can assume that $W$ is 3 -connected as $\operatorname{dim} W=n+1 \geq 8$. Consequently by the Hurewicz theorem we have $H_{i}(W)=0$ for all $i=1,2,3$ and $H_{4}(W) \cong \pi_{4}(W)$. Similarly, $H_{i}\left(M_{1}\right)=0$ for $i=1,2,3$ and $H_{4}\left(M_{1}\right) \cong \pi_{4}\left(M_{1}\right)$ since $M_{1}$ is 3 -connected.

Since for any 3 -connected space $X, H^{4}(X ; \mathbf{Z}) \cong \operatorname{Hom}\left(H_{4}(X ; \mathbf{Z}), \mathbf{Z}\right)$, the first Pontryagin class $p_{1}(W)$ is given by a homomorphism

$$
p_{1}(W): H_{4}(W ; \mathbf{Z}) \longrightarrow \mathbf{Z} .
$$

Similarly, the class $p_{1}\left(M_{1}\right)$ is given by a homomorphism $p_{1}\left(M_{1}\right): H_{4}\left(M_{1} ; \mathbf{Z}\right) \longrightarrow \mathbf{Z}$. Then $\left.T W\right|_{N} \cong T M_{1} \oplus \epsilon^{1}$, where $\epsilon^{1}$ is a trivial linear bundle, which implies that $p_{1}(W)=i^{*}\left(p_{1}\left(M_{1}\right)\right)$, where $i: M_{1} \hookrightarrow W$ is the boundary inclusion. Also recall that the first Pontryagin class is divisible by 2 for spin manifolds. Thus we obtain a commutative diagram:

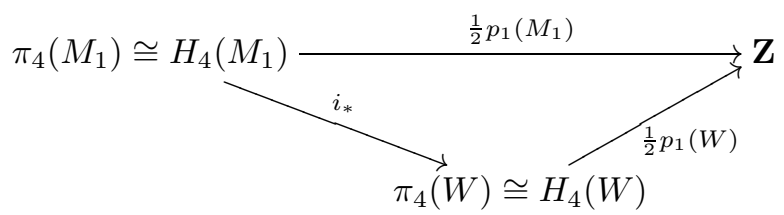

Remark. Let $H_{4}(W ; \mathbf{Z})=F_{4}(W) \oplus T_{4}(W)$, where $F_{4}(W)$ and $T_{4}(W)$ are free and torsion parts respectively. Clearly the homomorphism $p_{1}(W): H_{4}(W ; \mathbf{Z}) \longrightarrow \mathbf{Z}$, restricted to the torsion part $T_{4}(W)$, is trivial. Thus $p_{1}(W)$ is not a torsion class, and $p_{1}(W) / 2=0$ implies $p_{1}(W)=0$. 
Both manifolds $W$ and $M_{1}$ are 3 -connected and spin (where, of course, $M_{1} \subset$ $\partial W)$; however, they are not string manifolds, i.e. the first Pontryagin class is not zero. We would like to show that the kernel of the homomorphism $p_{1} / 2$ can be killed by surgeries.

Lemma 3.8. Let $\eta^{k} \rightarrow S^{4}$ be a vector bundle of dimension $k \geq 5$. Then there exists a 4-dimensional bundle $\xi^{4} \rightarrow S^{4}$ such that $\eta^{k} \cong \xi^{4} \oplus \epsilon^{k-4}$, where $\epsilon^{k-4} \rightarrow S^{4}$ is a trivial vector bundle.

Proof. Let $f: S^{4} \rightarrow B O(k)$ be a map classifying the bundle $\eta$. Then we can assume that $S^{4}$ is mapped to the 4-th skeleton $B O(k)^{(4)}$ of $B O(k)$. It is well known that $B O(k)^{(4)} \subset B O(4)$ if $k \geq 5$. Thus up to homotopy, the map $f$ factors through $B O(4)$; i.e. we obtain a commutative (up to homotopy) diagram

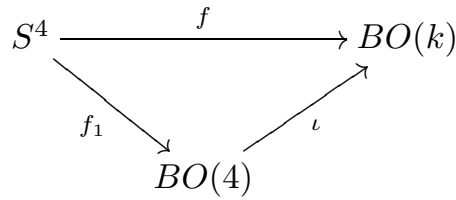

where $\iota: B O(4) \hookrightarrow B O(k)$ is the standard embedding. Hence $\eta^{k} \cong \xi^{4} \oplus \epsilon^{k-4}$.

We continue with the proof of Proposition 3.7. Let $S^{4} \hookrightarrow M_{1}$ be an embedded sphere representing an element $x \in \pi_{4}\left(M_{1}\right) \cong H_{4}\left(M_{1} ; \mathbf{Z}\right)$ such that $p_{1}(x)=0$. We denote by $\nu_{S^{4}}$ the normal of the embedding $S^{4} \hookrightarrow M_{1}$. By assumption, $\left.T M_{1}\right|_{S^{4}}$ is stably trivial, i.e. $\left.T M_{1}\right|_{S^{4}} \oplus \epsilon^{k-n} \cong \epsilon^{k}$ for some $k>n$. However, $\left.T M_{1}\right|_{S^{4}} \cong$ $T S^{4} \oplus \nu_{S^{4}}$, and we have that

$$
T S^{4} \oplus \nu_{S^{4}} \oplus \epsilon^{k-n} \cong \epsilon^{k} .
$$

Since $T S^{4} \oplus \epsilon^{1}$ is a trivial bundle,

$$
\epsilon^{5} \oplus \nu_{S^{4}} \oplus \epsilon^{k-n-1} \cong \epsilon^{k} .
$$

In particular, we obtain that $p_{1} \nu_{S^{4}}=0$, and Lemma 3.8 gives that $\nu_{S^{4}}=\xi^{4} \oplus \epsilon^{n-4}$, where $\xi^{4}$ is a 4 -dimensional bundle with $p_{1} \xi^{4}=0$. Thus $\xi^{4}$ is trivial bundle, i.e. the normal bundle $\nu_{S^{4}}$ is trivial. The same holds for an embedded sphere representing the kernel of the homomorphism $p_{1}(W): H_{4}(W ; \mathbf{Z}) \longrightarrow \mathbf{Z}$.

Thus we can use surgeries on $W$ to kill the kernel of $\frac{1}{2} p_{1}(W)$, and therefore the homomorphism $i_{*}: H_{4}\left(M_{1}\right) \rightarrow H_{4}(W)$ becomes an isomorphism onto its image. Therefore in the long exact sequence,

$$
H_{4}\left(M_{1}\right) \rightarrow H_{4}(W) \rightarrow H_{4}\left(W, M_{1}\right) \rightarrow H_{3}\left(M_{1}\right)
$$

the first map is onto and the last group is trivial. We conclude that $H_{4}\left(W, M_{1}\right)=0$. Therefore we obtain that $H_{i}\left(W, M_{1}\right)=0$ for all $i \leq 4$. Finally, Lemma 3.3 implies that $M_{1}$ can be obtained from $M_{0}$ by surgeries of codimension at least 5 .

3.2. Non-Fivebrane 7-connected manifolds. It is well known that the second Pontryagin class of a string manifold is divisible by 6 and $\frac{1}{6} p_{2}$ serves as the obstruction to lifting a string structure to a Fivebrane structure. One can without difficulties adapt the proof of Proposition 3.7 to show the following:

Proposition 3.9. Let $N$ be a 7-connected and non-Fivebrane compact manifold of dimension $\geq 15$. If $N$ is string cobordant to a manifold $M$, then $N$ can be obtained from $M$ by surgeries of codimension $\geq 9$. 


\section{Positive Curvature AND Surgeries}

The results of the previous section suggest that geometrical properties that are stable under surgeries should have a topological interpretation. This is the case for the positivity of the $p$-curvatures and the second Gauss-Bonnet curvature, as we will see in the rest of this paper.

4.1. Positive $p$-curvature. We denote by $G_{p}\left(\mathbf{R}^{n}\right)$ the Grassmanian manifold of $p$-dimensional subspaces in $\mathbf{R}^{n}$, as above. Let $(M, g)$ be a Riemannian manifold. Then the metric $g$ provides the tangent bundle $T M$ the structure group $O(n)$. This gives an associated smooth bundle

$$
G_{p}(T M):=T M \times_{O(n)} G_{p}\left(\mathbf{R}^{n}\right) \longrightarrow M,
$$

with the fiber $G_{p}\left(T M_{x}\right) \cong G_{p}\left(\mathbf{R}^{n}\right)$ over $x \in M$ and the structure group $O(n)$.

Then the $p$-curvature $s_{p}$, for $0 \leq p \leq n-2$, is a function

$$
s_{p}: G_{p}(T M) \longrightarrow \mathbf{R}
$$

defined as follows. Let $V$ be a tangent $p$-plane at $x \in M$. We choose an orthonormal basis $\left\{e_{i}\right\}$ of the orthogonal complement $V^{\perp}$ of $V$ in $T M_{x}$, and define

$$
s_{p}(V)=\sum_{i, j=p+1}^{n} K_{i, j},
$$

where $K_{i, j}=K\left(e_{i}, e_{j}\right)$ is the usual sectional curvature. The 0 -curvature $s_{0}$ coincides with the usual scalar curvature Scal, the 1-curvature is the Einstein curvature and the $(n-2)$-curvature is the usual sectional curvature.

We are interested in understanding the conditions under which a manifold admits a Riemannian metric $g$ with positive $p$-curvature.

We emphasize that if $s_{p}>$, then $s_{j}>0$ for all $j<p$. It turns out that the positivity of the $p$-curvature can be preserved under surgeries:

Theorem 4.1 ([8]). Let $g_{0}$ be a Riemannian metric on a compact manifold $M_{0}$ with $s_{p}\left(g_{0}\right)>0$, and $M_{1}$ be a manifold constructed out of $M_{0}$ by a surgery of codimension $\geq p+3$. Then there exists a Riemannian metric $g_{1}$ on $M_{1}$ with $s_{p}\left(g_{1}\right)>0$.

There is a natural generalization of Theorem 4.1 for elementary cobordism:

Theorem 4.2. Let $g_{0}$ be a Riemannian metric on a compact manifold $M_{0}$ with $s_{p}>0$ and let $M_{1}$ be a manifold constructed out of $M_{0}$ by a surgery of codimension $\ell+1 \geq p+3$. Let

$$
W=M_{0} \times I \cup\left(D^{k+1} \times D^{\ell+1}\right), \quad \partial W=M_{0} \sqcup M_{1},
$$

be the corresponding elementary cobordism. Then there exists a Riemannian metric $\bar{g}$ on $W$ with positive p-curvature and such that

$$
\begin{cases}\bar{g}=g_{0}+d t^{2} & \text { near } M_{0} \\ \bar{g}=g_{1}+d t^{2} & \text { near } M_{1}\end{cases}
$$

In particular, the p-curvature of the metric $g_{1}$ is positive.

Remark 4.3. The methods of M. Walsh's papers [21,22] can be adapted to provide a proof for the above Theorem 4.2 and also for Theorem 4.5 below. 
4.2. Positive second Gauss-Bonnet curvature. For a given Riemannian manifold $(M, g)$, we denote by $R$, Ric and Scal respectively the Riemann curvature tensor, the Ricci curvature tensor and the scalar curvature. The second GaussBonnet curvature, denoted by $h_{4}$, is a quadratic scalar curvature and is defined by

$$
h_{4}=\|R\|^{2}-\|\mathrm{Ric}\|^{2}+\frac{1}{4} \mathrm{Scal}^{2} ;
$$

see [11. Note that in four dimensions, the curvature $h_{4}$ coincides with the GaussBonnet integrand. This curvature is considered by physicists as a possible substitute to the usual scalar curvature to describe gravity in higher general theories of relativity, for instance in string theories. Here we are interested in the positivity properties of this invariant. First, let us recall the following stability under surgeries result:

Theorem 4.4 (11]). Let $g_{0}$ be a Riemannian metric on a compact manifold $M_{0}$ with $h_{4}\left(g_{0}\right)>0$, and $M_{1}$ be a manifold constructed out of $M_{0}$ by a surgery of codimension at least 5. Then there exists a Riemannian metric $g_{1}$ on $M_{1}$ with $h_{4}\left(g_{1}\right)>0$.

There is a natural generalization of Theorem 4.4 .

Theorem 4.5. Let $g_{0}$ be a Riemannian metric on a compact manifold $M_{0}$ with $h_{4}\left(g_{0}\right)>0$ and let $M_{1}$ be a manifold constructed out of $M_{0}$ by a surgery of codimension $\ell+1 \geq 5$. Let

$$
W=M_{0} \times I \cup\left(D^{k+1} \times D^{\ell+1}\right), \quad \partial W=M_{0} \sqcup M_{1},
$$

be the corresponding elementary cobordism. Then there exists a Riemannian metric $\bar{g}$ on $W$ with $h_{4}(\bar{g})>0$ and such that

$$
\begin{cases}\bar{g}=g_{0}+d t^{2} & \text { near } M_{0} \\ \bar{g}=g_{1}+d t^{2} & \text { near } M_{1} .\end{cases}
$$

In particular, $h_{4}\left(g_{1}\right)>0$.

Remarks 4.1. (1) Theorems 4.4 and 4.5 are still valid if we require the metric $g_{0}$ to have positive $h_{4}$ and positive 2 -curvature at the same time.

(2) Theorems 4.4 and 4.5 can also be generalized to all higher Gauss-Bonnet curvatures $h_{2 k}$. It is plausible that the condition $h_{2 k}>0$ could be preserved under surgeries of codimension at least $2 k+1$.

(3) Because $h_{4}$ is quadratic in the Riemann curvature tensor, one can expect that the condition $h_{4}>0$ has two components, where each component is an inequality that is linear in the Riemann curvature tensor. It would be interesting to determine these components. In this direction, Theorem 4.6 below relates the positivity of $h_{4}$ to the positivity and negativity of the p-curvatures.

Theorem 4.6 ([11]). Let $(M, g)$ be a Riemannian manifold of dimension $n \geq 4$. Assume that $s_{p}(g) \geq 0$ or $s_{p}(g) \leq 0$ (respectively, $s_{p}(g)>0$ or $s_{p}(g)<0$ ), where $p \geq \frac{n}{2}$. Then $h_{4}(g) \geq 0$ (respectively, $h_{4}(g)>0$ ). Furthermore, $h_{4}(g) \equiv 0$ if and only if the manifold $(M, g)$ is flat. 


\section{First applications: Proof of Theorems A, B and B'}

5.1. Proof of Theorem A. Theorem A is a consequence of the following theorem.

Theorem 5.1. Let $M_{1}$ be a compact 3-connected manifold of dimension $\geq g$ which is not string. If $M_{1}$ is spin cobordant to a manifold $M_{0}$ which carries a metric $g_{0}$ with $s_{2}\left(g_{0}\right)>0$ (respectively, with $h_{4}\left(g_{0}\right)>0$ ), then $M_{1}$ also carries a metric $g_{1}$ with $s_{2}\left(g_{1}\right)>0$ (respectively, with $\left.h_{4}\left(g_{1}\right)>0\right)$.

Proof. On one hand, Proposition 3.7 shows that the manifold $M_{1}$ can be obtained from $M_{0}$ by surgeries of codimension at least 5 . On the other hand, since $M_{0}$ has positive 2-curvature (resp. positive $h_{4}$-curvature), Theorems 4.1 and 4.4 therefore show that $M_{1}$ also carries a metric with $s_{2}>0$ (respectively, with $h_{4}>0$ ).

Now we prove Theorem A as follows. A compact 3-connected manifold $M$ of positive 2-curvature and with dimension $\geq g$ is in particular a simply connected manifold of positive scalar curvature and therefore its $\alpha$-genus vanishes by Theorem 1.2. Conversely, a compact non-string 3-connected manifold $M$ of dimension $\geq g$ and with vanishing $\alpha$-genus is spin cobordant to the total space $E$ of an $\mathbf{H P}^{2}$ bundle by Theorem 1.4. The total space $E$ has positive 2-curvature and positive $h_{4}$ curvature. Thus the above Theorem 5.1 shows that $M$ carries a metric with positive 2-curvature and a metric with $h_{4}>0$.

5.2. Proof of Theorems $\mathbf{B}$ and $\mathbf{B}^{\prime}$. Now we return to $B O\langle\ell\rangle$-manifolds. The following theorem unifies and generalizes at the same time Theorem B of [6], Lemma 4.2 of [8] and Theorems B and $\mathrm{B}^{\prime}$ of this paper that were stated in the introduction.

Theorem 5.2. Let $n, r, \ell$ be positive integers such that $n \geq 2 r+3$ and $\ell \geq r+2$. Let $M_{1}$ be a compact $r$-connected $B O\langle\ell\rangle$-manifold of dimension $n$. Assume $\left[M_{1}\right]=$ $\left[M_{0}\right]$ in the cobordism group $\Omega_{n}^{\langle\ell\rangle}$, where $M_{0}$ admits a Riemannian metric $g_{0}$ with $s_{p}\left(g_{0}\right)>0$ for some $p$ such that $0 \leq p \leq r-1$. Then $M_{1}$ also admits a Riemannian metric $g_{1}$ with $s_{p}\left(g_{1}\right)>0$.

Proof. Let $0 \leq p \leq r-1$ be as in the theorem. Proposition 3.4 asserts that the manifold $M_{1}$ can be obtained from $M_{0}$ using surgeries of codimensions $\geq r+2 \geq$ $p+3$. Since the manifold $M_{0}$ is supposed to have positive $p$-curvature, Theorem 4.1 shows that $M_{1}$ carries as well a metric with $s_{p}>0$.

Note that we recover Theorem B of [6] about the scalar curvature (that is, the 0 -curvature) when $p=0, r=1$ and $\ell=4$. Lemma 4.2 of [8] about the 1-curvature is obtained for $p=1, r=2$ and $\ell=4$. Theorems $\mathrm{B}$ and $\mathrm{B}^{\prime}$ of this paper are respectively obtained for $\ell=8$ and $\ell=9$.

Remarks 5.1. (1) Similar results hold if we replace positive 2-curvature by positive $h_{4}$-scalar curvature. For instance: A compact 3 -connected string manifold of dimension $n \geq 9$ that is string cobordant to a manifold of positive $h_{4}$ has a metric with positive $h_{4}$ curvature.

(2) Let $n=11$ or $n=13$. Since in these particular dimensions string $n$ manifolds are known to be cobordant to zero, we conclude that a compact 3 -connected string $n$-manifold always has a metric with positive 2 -curvature and a metric with $h_{4}$ positive. Similar results hold for the $p$-curvatures for $p \leq 5$ as above. 
5.3. Genera for string manifolds and positive curvature. Recall that the string cobordism ring $\Omega_{*}^{\text {string }}=\bigoplus_{n \geq 0} \Omega_{n}^{\text {string }}$ is the ring whose elements of order $n$ are string-cobordism classes of $n$-dimensional string manifolds, the addition operation is given by the disjoint union of manifolds and product operation is given by the Cartesian product of manifolds.

Let $I_{1}$ (resp. $I_{2}, I_{3}$ ) denote the subset of $\Omega_{*}^{\text {string }}$ which consists of bordism classes containing representatives with positive 2-curvature (resp. positive $h_{4}$, positive $h_{4}$ and positive $s_{2}$ ). Since the cartesian product of a manifold of positive 2-curvature (resp. positive $h_{4}$, positive $h_{4}$ and positive $s_{2}$ ) with an arbitrary manifold has positive 2-curvature (resp. positive $h_{4}$, positive $h_{4}$ and positive $s_{2}$ ), we conclude that $I_{1}$ (resp. $I_{2}, I_{3}$ ) is an ideal of $\Omega_{*}^{\text {string }}$. We therefore get the following three genera (ring homomorphisms):

$$
\Pi_{i}: \Omega_{*}^{\text {string }} \rightarrow \Omega_{*}^{\text {string }} / I_{i},
$$

for $i=1,2,3$. A natural question arises at this level: Are the previous three (geometrical) genera topological genera? Are they for instance related to Witten genus?

Recall that for a string manifold $N$, the Witten genus, denoted $\phi_{W}(N)$, is a modular form for $S L_{2}(\mathbf{Z})$ with integer coefficients. In particular, the Witten genus $\phi_{W}$ defines a ring homomorphism from the bordism ring $\Omega_{*}^{\text {string }}$ to the ring of integral modular forms for $S L_{2}(\mathbf{Z})$; see the next section for more details.

We shall prove in section 7 that $\operatorname{Ker} \phi_{W} \otimes \mathbf{Q} \subset I_{i}$ for $i=1,2,3$. It remains an open question to decide whether the previous inclusion is in fact an equality, that is, a vanishing theorem of Lichnérowicz type: If $N$ is a string manifold of positive 2-curvature (resp. positive $h_{4}$, positive $h_{4}$ and positive $s_{2}$ ), then $\phi_{W}(N)=0$.

An important question in the same direction is Stolz's conjecture [16]:

Stolz conjecture (1996). If $N$ is a string manifold and admits a positive Ricci curvature metric, then $\phi_{W}(N)=0$.

At this time no counterexamples are known to this conjecture, and the conjecture is proven to be true for some classes. However, these classes also admit metrics with different positivity properties, for instance metrics with positive $p$-curvature, and so it may be possible that the Stolz conjecture is true for positive $p$-curvature.

Let us note here that all the known constructions of string manifolds with positive $p$-curvature through group actions and Riemannian submersions [10] or through surgeries [8] have vanishing Witten genus. This is a consequence of a result due to Dessai, Höhn and Liu [5, 12, where they prove the vanishing of the Witten genus on connected string manifolds with non-trivial smooth $S^{3}$-actions and of another related result of Dessai [5]. The latter asserts the vanishing of the Witten genus on any smooth fiber bundle of closed oriented manifolds provided the fiber is a string manifold and the structure group is a compact connected semi-simple Lie group which acts smoothly and non-trivially on the fiber.

\section{Witten Genus AND ITS KeRnel IN $\Omega_{*}^{\text {string }} \otimes \mathbf{Q}$}

6.1. Cayley projective plane. Here we recall necessary facts concerning the Cayley projective plane $\mathbb{C a P}^{2}$. We follow the constructions due to A. Dessai [5]. Let $F_{4}$ denote the 52-dimensional compact simple sporadic Lie group. It is well known 
that $F_{4}$ contains a group isomorphic to $\operatorname{Spin}(9)$ which is unique up to inner automorphism of the ambient group $F_{4}$. We choose such a subgroup and identify it by $\operatorname{Spin}(9)$. Then we can identify the Cayley projective plane $\mathbb{C a P}^{2}$ with the homogeneous space $F_{4} / \operatorname{Spin}(9)$. This is a 7 -connected smooth manifold with the cohomology ring $H^{*}\left(\mathbb{C a P}^{2} ; \mathbf{Z}\right) \cong \mathbf{Z}[z] / z^{3}$, where $z \in H^{8}\left(\mathbb{C a P}^{2} ; \mathbf{Z}\right)$ is a generator. In particular, $\mathrm{CaP}^{2}$ is a fiber of the fiber bundle

$$
\operatorname{BSpin}(9) \rightarrow \mathrm{BF}_{4}
$$

induced by the embedding $\operatorname{Spin}(9) \subset F_{4}$. The bundle $B \operatorname{Spin}(9) \rightarrow B F_{4}$ is a universal geometric $\mathbb{C a P}^{2}$-bundle.

Let $L$ be a smooth manifold, $\operatorname{dim} L=\ell$, and $f: L \rightarrow B F_{4}$ be a map. Then one obtains the induced bundle with the fiber $\mathbb{C a P}^{2}$ and structure group $F_{4}$ :

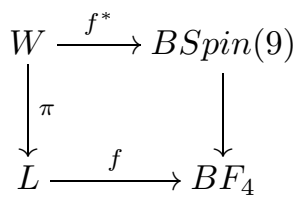

Let $T_{\text {spin }} \subset \operatorname{Spin}(9)$ be the maximal torus covering the maximal torus $T_{\text {so }} \subset S O(9)$. It is convenient to choose a basis $\hat{\xi}_{1}, \hat{\xi}_{2}, \hat{\xi}_{3}, \hat{\xi}_{4}$ of the Lie algebra of $T_{\text {so }}$ which is also a basis for the Lie algebra of $T_{\text {spin }}$. Then the integral lattice in $\mathbf{R}^{4}$ which provides the universal cover of the torus $T_{\text {spin }}$ is given by $a_{1} \hat{\xi}_{1}+\cdots+a_{4} \hat{\xi}_{4}$, where the sum $a_{1}+\cdots+a_{4}$ of integers is even. Let $\hat{\xi}$ be a generator of the Lie algebra of $S^{1}$ and $v: S^{1} \rightarrow T_{\text {spin }}$ be such a map for which the differential $d v$ takes $\hat{\xi}$ to $2 \hat{\xi}_{1}$. Then the composition

$$
\hat{v}: S^{1} \stackrel{v}{\longrightarrow} T_{\text {spin }} \longrightarrow \operatorname{Spin}(9) \longrightarrow F_{4}
$$

induces a map $B \hat{v}: B S^{1} \rightarrow B F_{4}$. We obtain the following diagram of fiber bundles:

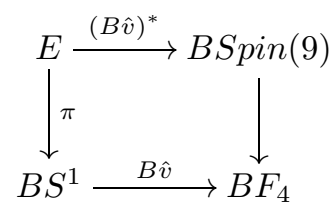

where the bundle $\pi: E \rightarrow B S^{1}$ has the fiber $\mathbb{C a P}^{2}$ and the structure group is reduced from the group $F_{4}$ to its subgroup $v\left(S^{1}\right) \subset F_{4}$.

Then one can choose a subgroup $S^{3}$ of the centralizer of the group $v\left(S^{1}\right)$ in $F_{4}$ so that $S^{3}$ acts non-trivially on the orbit space $\mathbb{C a P}^{2}=F_{4} / \operatorname{Spin}(9)$. A particular choice is given by the subgroup $S^{3} \cong \operatorname{Spin}(3) \subset \operatorname{Spin}(9)$ which covers the subgroup

$$
\left(\begin{array}{c|c}
1 & 0 \\
\hline 0 & S O(3)
\end{array}\right) \subset S O(9)
$$

under the canonical covering map $\operatorname{Spin}(9) \rightarrow S O(9)$. Then the subgroup $S^{3}$ commutes with the structure group $v\left(S^{1}\right)$ of the fiber bundle $\pi: E \rightarrow B S^{1}$. Thus we obtain a non-trivial action of $S^{3}$ along the fibers $\mathbb{C a P}^{2}=F_{4} / \operatorname{Spin}(9)$ of the total space $E$. 
Assume that a map $f: L \rightarrow B F_{4}$ is given by a composition $L \stackrel{h}{\longrightarrow} B S^{1} \stackrel{B v}{\longrightarrow} B F_{4}$. Then the geometric $\mathbb{C a P}^{2}$-bundle $W \rightarrow L$ is given by the diagram of fiber bundles

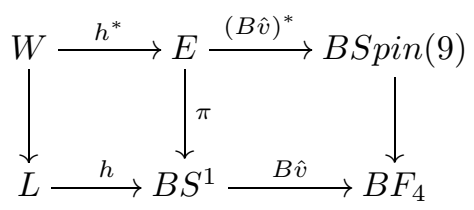

In particular, the structure group of the bundle $W \rightarrow L$ is reduced to $v\left(S^{1}\right) \subset F_{4}$, and there is a non-trivial fiber-wise action of $S^{3}$ on $W$. This construction leads to the following result:

Proposition 6.1 (A. Dessai, 5, Proposition 5.2]). There exist oriented manifolds $M^{4 k}$ such that

$$
\Omega_{*}^{\mathrm{so}} \otimes \mathbf{Q} \cong \mathbf{Q}\left[x_{4}, \ldots, x_{4 k}, \ldots\right],
$$

where $x_{4 k}=\left[M^{4 k}\right]_{\mathrm{so}}, k=1,2, \ldots$, and $M^{4 k}$ satisfy the following conditions:

(a) $M^{4 k}$ is a simply connected spin manifold for all $k \geq 1$;

(b) $M^{4 k}$ is a string manifold for all $k \geq 2$;

(c) $M^{4 k}$ is the total space of a geometric $\mathbb{C a P}^{2}$-bundle with structure group $S^{1}$ and non-trivial $S^{3}$-action along the fibers for $k \geq 4$.

We recall key points on the construction of manifolds $M^{4 k}$ given by A. Dessai 5 . The manifold $M^{4}$ can be chosen as the $K_{3}$-surface given by the quartic $x_{0}^{4}+\cdots+x_{3}^{4}=$ 0 in $\mathbf{C P}^{3}$, and the manifolds $M^{8}$ and $M^{12}$ as almost parallelizable manifolds with non-vanishing top Pontyagin class. These manifolds can be constructed by means of plumbing; see [7]. The manifold $M^{16}=\mathbb{C a P}^{2}$ is the Cayley projective plane. For $k \geq 5$, the manifold $M^{4 k}$ can be chosen as the total space of the geometric $\mathbb{C a P}^{2}$ bundle over a complete intersection $L_{k-4} \subset \mathbf{C P}^{2 k-4}$ of complex dimension $2 k-8$. The manifold $L_{k-4}$ comes together with a non-trivial class $c \in H^{2}\left(L_{k-4} ; \mathbf{Z}\right)$ which is the first Chern class of the restriction of the dual Hopf bundle over $\mathbf{C P}^{2 k-4}$. Then for a carefully chosen integer $a_{k-4}$, the class $a_{k-4} c \in H^{2}\left(L_{k-4} ; \mathbf{Z}\right)$ gives a map $h_{k}: L_{k-4} \rightarrow B S^{1}$ such that the induced bundle $W \rightarrow L_{k-4}$ given by (6.1), where we let $L=L_{k-4}$ and $h=h_{k}$, is a geometric $\mathbb{C a P}^{2}$-bundle with structure group $S^{1}$ and non-trivial $S^{3}$-action along the fibers.

Let $M$ be a spin manifold, $\operatorname{dim} M=4 k$. Then the $\hat{A}(M)$ is well defined and coincides with the index of the standard Dirac operator on $M$. For any real vector bundle $V$ over $M$, we denote by $\hat{A}(M ; V)$ the index of the Dirac operator on $M$ twisted by the complexified vector bundle $V \otimes \mathbf{C}$. A total symmetric power $S_{t}(V)$ of a vector bundle $V$ is given as a series

$$
S_{t}(V):=1+S^{1}(V) t+S^{2}(V) t^{2}+\cdots,
$$

where $S^{j}(V)$ is the $j$-th symmetric power of $V$ and $t$ is an indeterminate variable. Consider the tensor product

$$
\mathbb{S}(V):=\bigotimes_{m=1}^{\infty} S_{q^{m}}(V)=1+V q+\left(S^{2}(V) \oplus V\right) q^{2}+\left(S^{3}(V) \oplus(V \otimes V) \oplus V\right) q^{3}+\cdots
$$


see [19, Section 2]. Then the Witten genus $\phi_{W}(M)$ (where $\operatorname{dim} M=4 k$ ) is defined as the series

$$
\begin{aligned}
\phi_{W}(M) & =\hat{A}(M ; \mathbb{S}(V)) \cdot \prod_{n=1}^{\infty}\left(1-q^{n}\right)^{4 k} \\
& =\left(1+V q+\left(S^{2}(V) \oplus V\right) q^{2}+\cdots\right) \cdot \prod_{n=1}^{\infty}\left(1-q^{n}\right)^{4 k}
\end{aligned}
$$

see [19, Section 2] or [5, Section 2]. It is easy to see that $\phi_{W}(M) \in \mathbf{Z}[[q]]$ and $\hat{A}(M)$ is the constant term of the series $\phi_{W}(M)$. In particular, the Witten genus defines the homomorphism

$$
\phi_{W}: \Omega_{*}^{\text {string }} \longrightarrow \mathbf{Z}[[q]] .
$$

Proposition 6.1 implies the following result

Corollary 6.2 (A. Dessai, [5]). (1) There is an isomorphism $\Omega_{*}^{\text {string }} \otimes \mathbf{Q} \cong$ $\mathbf{Q}\left[x_{8}, x_{12}, x_{16}, \ldots, x_{4 k}, \ldots\right]$, where $x_{4 k}=\left[M^{4 k}\right]_{\text {string }}$, and the string manifolds $M^{4 k}$ are as in Proposition 6.1

(2) The kernel $\left(\operatorname{Ker} \phi_{W}\right) \otimes \mathbf{Q} \subset \Omega_{*}^{\text {string }} \otimes \mathbf{Q}$ coincides with the ideal generated by the elements $x_{4 k}, k \geq 4$.

(3) If $x \in \operatorname{Ker} \phi_{W} \subset \Omega_{*}^{\text {string }}$, then some multiple of $x$ can be realized as the total space of a geometric $\mathbb{C a P}^{2}$-bundle.

We emphasize that the string manifolds $M^{8}$ and $M^{12}$ have non-trivial Witten genus just because $\hat{A}\left(M^{8}\right)$ and $\hat{A}\left(M^{12}\right)$ are non-zero by construction.

\section{Further applichtions: Proof of Theorem A' And Corollary B}

Corollary 6.2 asserts in particular that if $N$ is a string manifold with vanishing Witten genus, then a non-zero multiple of $N$ is string cobordant to a string manifold which is the total space of a $\mathbb{C a P}{ }^{2}$-bundle with structure group $S^{1}$ and non-trivial $S^{3}$-action along the fibers.

On the other hand, the Cayley projective plane $\mathbb{C a P}^{2}$ has dimension 16 and positive sectional curvature. In particular, using a result of 10, the total spaces of $\mathbb{C a P}^{2}$ bundles have positive $p$-curvature for $0 \leq p \leq 14$ (and as well positive $h_{4}$-curvature). Corollary B therefore immediately results from Theorem B.

Next, we prove Theorem A'. Let $N$ be a 7 -connected and non-Fivebrane compact manifold of dimension $\geq 15$. Assume that $N$ is string-cobordant to a manifold $M$ which carries a metric with positive 6 -curvature. Proposition 3.9 shows that the manifold $N$ can then be obtained by performing surgeries on $M$ of codimension $\geq 9 \geq 6+3$. Theorem 4.1 then implies that $N$ carries a metric of positive 6 curvature.

Finally, the manifold $N$ is 7-connected, so it is a string manifold. Since the Witten genus of $N$ is zero, by Corollary 6.2 a non-zero multiple of $N$ is string cobordant to a string manifold which is the total space of a $\mathbb{C a P}^{2}$-bundle. As above the total spaces of $\mathbb{C a P}^{2}$-bundles have positive 6 -curvature. We therefore deduce from the first part of this theorem that some multiple $N \sharp \cdots \sharp N$ carries a metric of positive 6-curvature. 


\section{ACKNOWLEDGEMENTS}

The authors would like to thank the referee for the careful reading of the paper and for useful comments. The first author thanks Michael Joachim and Anand Dessai and IHĖS for partial financial support. The second author acknowledges financial support from the Deanship of Scientific Research at the University of Bahrain (ref. 2011/13).

\section{REFERENCES}

[1] Bernd Ammann, Mattias Dahl, and Emmanuel Humbert, Surgery and harmonic spinors, Adv. Math. 220 (2009), no. 2, 523-539, DOI 10.1016/j.aim.2008.09.013. MR2466425 (2009h:58050)

[2] M. Ando, M. J. Hopkins, and N. P. Strickland, Elliptic spectra, the Witten genus and the theorem of the cube, Invent. Math. 146 (2001), no. 3, 595-687, DOI 10.1007/s002220100175. MR,1869850(2002g:55009)

[3] Matthew Ando, Michael J. Hopkins, and Neil P. Strickland, The sigma orientation is an $H_{\infty}$ map, Amer. J. Math. 126 (2004), no. 2, 247-334. MR2045503 (2005d:55009)

[4] Christian Bär and Mattias Dahl, Surgery and the spectrum of the Dirac operator, J. Reine Angew. Math. 552 (2002), 53-76, DOI 10.1515/crll.2002.093. MR1940432 (2003h:58041)

[5] Anand Dessai, Some geometric properties of the Witten genus, Alpine perspectives on algebraic topology, Contemp. Math., vol. 504, Amer. Math. Soc., Providence, RI, 2009, pp. 99115, DOI 10.1090/conm/504/09877. MR2581907 (2012c:58036)

[6] Mikhael Gromov and H. Blaine Lawson Jr., The classification of simply connected manifolds of positive scalar curvature, Ann. of Math. (2) 111 (1980), no. 3, 423-434, DOI 10.2307/1971103. MR577131 (81h:53036)

[7] John W. Milnor and Michel A. Kervaire, Bernoulli numbers, homotopy groups, and a theorem of Rohlin, Proc. Internat. Congress Math. 1958, Cambridge Univ. Press, New York, 1960, pp. 454-458. MR0121801(22 \#12531)

[8] Mohammed-Larbi Labbi, Stability of the p-curvature positivity under surgeries and manifolds with positive Einstein tensor, Ann. Global Anal. Geom. 15 (1997), no. 4, 299-312, DOI 10.1023/A:1006553611999. MR1472322 (98e:53065)

[9] M.-L. Labbi, Compact manifolds with positive Einstein curvature, Geom. Dedicata 108 (2004), 205-217, DOI 10.1007/s10711-004-0898-0. MR2112675(2005i:53041)

[10] Mohammed-Larbi Labbi, Actions des groupes de Lie presque simples et positivité de la pcourbure (French, with English and French summaries), Ann. Fac. Sci. Toulouse Math. (6) 6 (1997), no. 2, 263-276. MR.1611832 (99e:53052)

[11] Mohammed-Larbi Labbi, Manifolds with positive second Gauss-Bonnet curvature, Pacific J. Math. 227 (2006), no. 2, 295-310, DOI 10.2140/pjm.2006.227.295. MR2263019|(2008a:53034)

[12] Kefeng Liu, Modular forms and topology, Moonshine, the Monster, and related topics (South Hadley, MA, 1994), Contemp. Math., vol. 193, Amer. Math. Soc., Providence, RI, 1996, pp. 237-262, DOI 10.1090/conm/193/02374. MR/1372725 (97f:58124)

[13] Rafe Mazzeo, Daniel Pollack, and Karen Uhlenbeck, Connected sum constructions for constant scalar curvature metrics, Topol. Methods Nonlinear Anal. 6 (1995), no. 2, 207-233. MR 1399537 (97e:53076)

[14] Jimmy Petean, The Yamabe invariant of simply connected manifolds, J. Reine Angew. Math. 523 (2000), 225-231, DOI 10.1515/crll.2000.049. MR:1762961(2001g:53075)

[15] Andrew Ranicki, Algebraic and geometric surgery, Oxford Mathematical Monographs, The Clarendon Press Oxford University Press, Oxford, 2002. Oxford Science Publications. MR2061749(2005e:57075)

[16] Hisham Sati, Urs Schreiber, and Jim Stasheff, Fivebrane structures, Rev. Math. Phys. 21 (2009), no. 10, 1197-1240, DOI 10.1142/S0129055X09003840. MR2588823(2011f:53045)

[17] R. Schoen and S. T. Yau, On the structure of manifolds with positive scalar curvature, Manuscripta Math. 28 (1979), no. 1-3, 159-183, DOI 10.1007/BF01647970. MR.535700 (80k:53064)

[18] Stephan Stolz, Simply connected manifolds of positive scalar curvature, Ann. of Math. (2) 136 (1992), no. 3, 511-540, DOI 10.2307/2946598. MR.1189863 (93i:57033) 
[19] Stephan Stolz, A conjecture concerning positive Ricci curvature and the Witten genus, Math. Ann. 304 (1996), no. 4, 785-800, DOI 10.1007/BF01446319. MR1380455 (96k:58209)

[20] David Wraith, Surgery on Ricci positive manifolds, J. Reine Angew. Math. 501 (1998), 99113, DOI 10.1515/crll.1998.082. MR.1637825(99j:53050)

[21] Mark Walsh, Metrics of positive scalar curvature and generalised Morse functions, Part I, Mem. Amer. Math. Soc. 209 (2011), no. 983, xviii+80, DOI 10.1090/S0065-9266-10-00622-8. MR2789750(2012c:53049)

[22] Mark Walsh, Metrics of positive scalar curvature and generalised Morse functions, Part II, Trans. Amer. Math. Soc. 366 (2014), no. 1, 1-50. MR3118389

[23] J. Wolf, Spaces of constant curvature (Fourth edition), Publish or Perish, Houston, 1977.

Department of Mathematics, University of Oregon, Eugene, Oregon 97403-1222

E-mail address: botvinn@math.uoregon.edu

Department of Mathematics, College of Science, University of Bahrain, 32038, BAHRAIN

E-mail address: labbi@sci.uob.bh 